\title{
Soybean Fermented with Bacillus amyloliquefaciens (Cheonggukjang) Ameliorates Atopic Dermatitis-Like Skin Lesion in Mice by Suppressing Infiltration of Mast Cells and Production of IL-31 Cytokine
}

\author{
Byoung Ok Cho ${ }^{1,2}$, Jae Young Shin ${ }^{1,3}$, Ji-su Kim ${ }^{1,3}$, Denis Nchang Che ${ }^{1,4}$, Hyun Ju Kang ${ }^{2}$, Do-Youn Jeong ${ }^{5}$, and \\ Seon Il Jang ${ }^{1,2 *}$ \\ ${ }^{1}$ Department of Health Management, Jeonju University, Jeonju 55069, Republic of Korea \\ ${ }^{2}$ Research Institute, Ato QEA Co. Ltd, Jeonju 54840, Republic of Korea \\ ${ }^{3}$ Department of Agro-Bio and Food Industry, Jeonju University, Jeonju 55069, Republic of Korea \\ ${ }^{4}$ Department of Food Science and Technology, Chonbuk National University, Jeonju 54896 Republic of Korea \\ ${ }^{5}$ Microbial Institute for Fermentation Industry (MIFI), Sunchang-gun, Jeonbuk 56048, Republic of Korea
}

Received: December 22, 2018

Revised: April 9, 2019

Accepted: April 10, 2019

First published online April 12, 2019

${ }^{*}$ Corresponding author Phone: +82-63-220-3124; Fax: +82-63-220-2054;

E-mail: sonjjang@jj.ac.kr

pISSN 1017-7825, eISSN 1738-8872

Copyright@ 2019 by

The Korean Society for Microbiology and Biotechnology
The present study was conducted with the aim to investigate the ameliorative effects of a new soybean product (cheonggukjang) fermented with Bacillus amyloliquefaciens SCGB1 (SFBA) in atopic dermatitis (AD) mouse model. Visual evaluation of AD induction in the mice indicated the remarkable control of SFBA in reducing the pathological severity of AD-like skin lesions reported as the SCORAD score of AD clinical symptoms. The results revealed that SFBA reduced dorsal skin and epidermal thickness to a similar extent with prednisolone. Further analysis revealed the dominance of SFBA in restraining mast cell infiltration in the dermis; immunoglobulin-E expression in serum; and TH2 IL-4 cytokine and itch-related IL-31 cytokine in the mice skin and serum. SFBA also suppressed scratching behaviours in mice induced by compound 48/80. Further histological findings also revealed the alleviation of collagen fiber deposition in dermal skin of the AD mice model. These actions of SFBA were examined to be mediated by its suppression of the phosphorylation activation of key signalling molecules such as NF- $\mathrm{B}$ and MAPK responsible for the induction of cytokine production. Thus, SFBA can be considered as a promising functional food for managing clinical, histological and immunological spectra associated with AD.

Keywords: Cheonggukjang, Bacillus amyloliquefaciens SCGB1, atopic dermatitis, scratching behaviour, IL-31, collagen

\section{Introduction}

Atopic dermatitis (AD), an allergic inflammatory disease characterized by intense pruritus, relapsing inflammation, and chronic eczematous plaques is caused by either an inside-out hypothesis mediated by Immunoglobulin $\mathrm{E}$ ( $\operatorname{IgE}$ ) or an outside-in hypothesis whereby disruption of the skin barrier (due to intrinsic genetic defect or environmental alteration) lead to sensitization and atopic disease [1]. AD is a leading cause of discomfort and distress in developed countries affecting up to $20 \%$ of children, the majority of whom outgrow the disease within a few years. Also, an estimated $2-9 \%$ of adults are reported to be suffering from the disease [2]. In $\mathrm{AD}$, infiltration of T-cells particularly skewed towards the $\mathrm{T}$ helper 2 (TH2) differentiation produces cytokines such as interleukin (IL)-4, IL-13, and IL-31 [3, 4]. IL-4 and IL-13 down-regulate the expressions of filaggrin, loricrin, and involucrin, three components of the skin involved in skin barrier integrity $[5,6]$. In addition, IL-4 and IL-13 induce B-cell antibody class switching to 
IgE, which is a key initiator of allergic diseases. The function of $\operatorname{IgE}$ in the development of $\mathrm{AD}$ is supported by the beneficial effect of anti-IgE therapy in a number of clinical studies [7]. IL-31 is a recently discovered cytokine that is strongly implicated in playing a key role in the development of itch in the AD in humans and mice models $[8,9]$. Another adverse situation in chronic AD which has been described in detail elsewhere is hyperkeratosis and type I collagen deposition, caused by thymic stromal lymphopoietin (TSLP) and Eosinophil-derived leukotriene $\mathrm{C} 4[10,11]$. The activation and translocation of NF- $\mathrm{B}$ into the nucleus and the activation of mitogen-activated protein kinase (MAPK) signaling pathways have also been implicated in the pathogenesis of $\mathrm{AD}$ by triggering the gene and protein expression of AD-related cytokines and chemokines [12-14]. Thus, drugs that interfere with NF-кB and MAPK activities may provide an alternative therapeutic strategy for the treatment of AD.

Nowadays, the use of topical corticosteroids for the treatment of $\mathrm{AD}$ poses risks of systemic and local side effects such as skin atrophy, solar purpura, stretch marks, increased hair thickness, muscle weakness, headache, and high blood pressure. As a result, many studies have explored the possibility of using novel therapeutic alternatives especially from natural products with fewer side effects for the treatment of AD. Some of these studies are focused on the use of fermented natural products, as such products contain numerous enzymes, microorganisms and bioactive compounds that are absent in the unfermented products [15]. One such study reported that during fermentation with a mold, flavonoid glycosides of soybean are converted into aglycones by hydrolysis which possesses biological functions [16]. In addition, soybeans fermented with different bacteria strains have been shown to possess biological activities spanning through anti-obesity, antidiabetic and anti-inflammatory effects in a number of chronic human diseases and mice studies [17-19]. Choi et al. (2008) showed that the Korean traditional fermented soybean food (cheonggukjang) exhibits its anti-inflammatory activity by reducing leukotriene production in lipopolysaccharide-treated RAW 264.7 cells and also by reducing passive cutaneous anaphylaxis and arachidonic acid-induced ear edema in rats, thus suggesting that cheonggukjang may be beneficial for several allergic conditions such as asthma and atopic dermatitis [20]. However, this hypothesis has not been proven yet. Therefore, in the present study, we sought to investigate whether oral administration of this soybean product (cheonggukjang) fermented with Bacillus amyloliquefaciens SCGB1 significantly ameliorated AD symptoms in a mice model of atopic dermatitis and to demonstrate its mechanism of action.

\section{Materials and Methods}

\section{Chemicals}

Fluoro-2,4-dinitrobenzene (DNFB), compound 48/80, Eosin, haematoxylin, and toluidine blue were purchased from SigmaAldrich (USA). House dust mite antigen came from Biostir (Japan) and a Trichrome Staining Kit was obtained from Abcam, Inc. (UK). IL-4 and IgE ELISA kits came from R\&D Systems (USA) and Shibayagi Co. (Japan), respectively. Antibodies against NF-кB

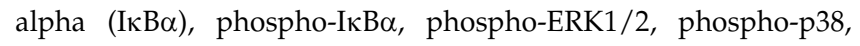
phospho-c-Jun N-terminal kinases (JNK), and phospho-NF-kB/ p65 were purchased from Santa Cruz Biotechnology (USA). Antibodies against IL-31 came from Thermo Scientific (USA) and Abcam. Antibodies against tryptase were purchased from Abcam while antibodies against actin came from Biosciences (USA).

\section{Cheonggukjang Preparation}

The soybeans used in the study were purchased from a local market in Sunchang-gun, Jeollabuk-do, Republic of Korea. B. amyloliquefaciens SCGB1 (KCCM11964P) used for the fermentation of the soybeans came from the Microbial Institute for Fermentation Industry (Korea). The soybeans (100 g) were washed and soaked with four volumes of distilled water at room temperature for $24 \mathrm{~h}$, after which they were autoclaved for $30 \mathrm{~min}$ at $121^{\circ} \mathrm{C}$. Thereafter, the soybeans were allowed to cool to under $40^{\circ} \mathrm{C}$. Next, the soybeans were inoculated with $5 \%(\mathrm{v} / \mathrm{w})$ of B. amyloliquefaciens $\left(1 \times 10^{9}\right.$ cells $\left./ \mathrm{ml}\right)$ and fermented for $24 \mathrm{~h}$ at $37^{\circ} \mathrm{C}$ and at a humidity of $80 \%$. The fermented soybeans (hereafter named SFBA) were freeze-dried, homogenized, and the powder was stored at $-80^{\circ} \mathrm{C}$ for future use.

\section{Animals and Experimental Design}

Seven-week-old, male, hairless mice, from Orient Bio Inc. (Korea), were used in this study. The mice were housed in a room with standard environmental conditions of temperature $-22 \pm 2{ }^{\circ} \mathrm{C}$, humidity $50-60 \%$, and a $12 / 12 \mathrm{~h}$ light-dark cycle and fed with commercial standard laboratory diet and water ad libitum. The experimental procedures were performed following the Jeonju University Institutional Animal Care and Use Committee guidelines (Approval No. JJU-IACUC-2018-9). After one week of initial acclimatization, the mice were divided into 4 groups $(n=5)$ i.e. Group 1-Normal control; Group 2-DNFB + house dust mite antigen (AD model); Group 3- AD model + SFBA; Group 4- AD model + prednisolone. To induce AD-like symptoms and skin lesions in groups 2,3, and 4, the dorsal skin of the mice in each of the groups was sensitized by repeated application of $0.1 \mathrm{ml}$ of $0.15 \%$ DNFB (prepared with acetone/olive oil in the ratio of 3:1) once on days 1 and 4 . On days 7,10 , and 13, the sensitized mice were challenged with $0.1 \mathrm{ml}$ of $0.2 \%$ DNFB and $100 \mathrm{mg}$ of dust mite antigen on the dorsal skin. The mice in the control group 
A
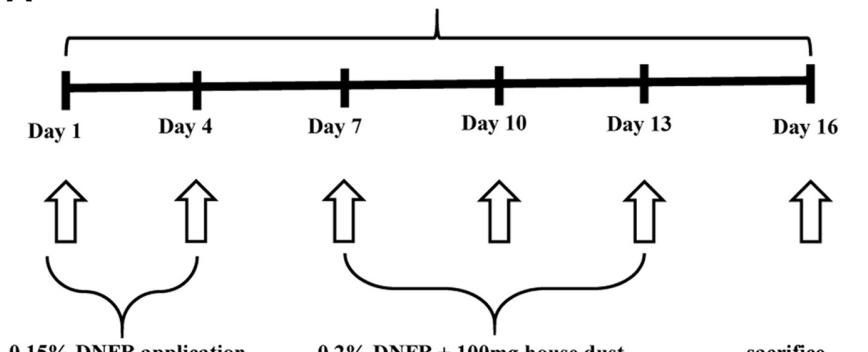

$0.2 \%$ DNFB + 100mg house dust mite antigen application

B
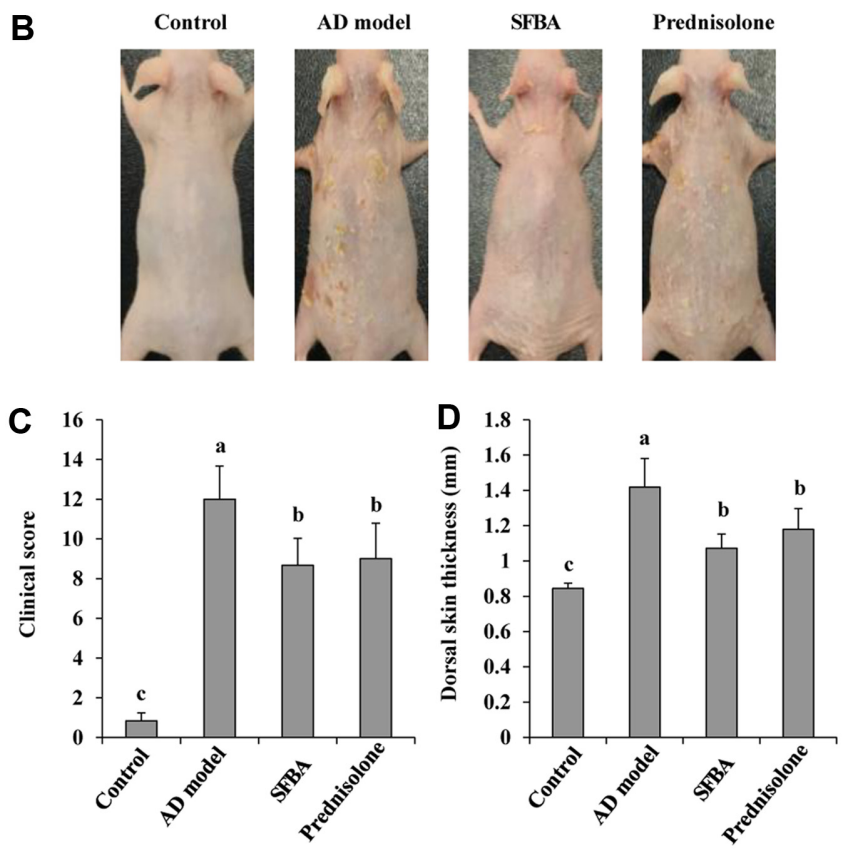

Fig. 1. Effect of SFBA treatment on the severity of clinical scores in hairless mice.

(A) Schematic diagram showing the experimental design for atopic dermatitis induction and treatment method. (B) Representative dorsal skin photographs were taken at the end of the experiment showing a comparison of AD-like skin lesions. (C) Clinical score of AD-like skin lesions in hairless mice was evaluated macroscopically. The dermatitis score was determined from the sum of all individual scores. (D) Dorsal skin thickness was measured with the help of a digital caliper. Data are presented as the mean $\pm \mathrm{SD}(n=5)$. Bars with different letters indicate statistically significant difference at $p<0.05$.

(Group 1) were applied with the same volume of vehicle (acetone/olive oil). Groups 1 and 2 mice were treated with saline while Groups 3 and 4 were treated with SFBA $400 \mathrm{mg} / \mathrm{kg}$, and prednisolone $10 \mathrm{mg} / \mathrm{kg}$, respectively. Treatment was done once daily throughout the entire period of the experiment and all mice received an equal volume $(0.1 \mathrm{ml})$ of saline, SFBA or prednisolone (Fig. 1A).
Four-week-old male ICR mice were obtained from the Orient Bio Inc. (Korea). The mice were housed and fed with similar conditions as above. After one week of initial acclimatization, they were divided into 4 Groups $(n=5)$ i.e. Group 1-Normal control; Group 2- compound 48/80; Group 3- compound 48/80 + SFBA; Group 4- compound $48 / 80+$ prednisolone. To induce pruritus in groups 2,3 , and $4,50 \mu \mathrm{g} /$ site $(0.1 \mathrm{ml})$ of compound $48 / 80$ was subcutaneously injected into the left side of the shoulder. Groups 2, 3, and 4 were treated with saline, SFBA $400 \mathrm{mg} / \mathrm{kg}$ and prednisolone $10 \mathrm{mg} / \mathrm{kg}$ respectively, $60 \mathrm{~min}$ before the injection of the compound 48/80. The mice in Group 1 were equally administered with saline. Thereafter, according to a previously published method [21], scratching behavior was monitored with micro-cameras (ONCCTV, Korea) for $60 \mathrm{~min}$ and the number of scratches on the site of the compound $48 / 80$ injections was counted in a double-blinded manner.

\section{Dermatitis Score}

The severities of AD-like symptoms were evaluated macroscopically by SCORAD method [22], which evaluates the degree of dryness, erosion, excoriation, and scaling. The overall dermatitis score was determined from the sum of all individual scores in a double-blinded manner. The degree of each symptom was graded from 0 (none), 1 (mild), 2 (moderate) and 3 (severe). Skin thickness was measured with the help of a digital caliper (Japan).

\section{Histopathological Analysis}

On day 16, all mice were sacrificed and the dorsal skins were harvested. The dorsal skin sections of the ICR mice injected with compound 48/80 were also harvested. The tissues were fixed in $4 \%$ paraformaldehyde in phosphate buffered saline (PBS, pH 7.4) for $24 \mathrm{~h}$; washed in PBS for $24 \mathrm{~h}$ with the PBS changed 5 times; dehydrated in a series of graduated ethanol (60-100\%); cleared in xylene and finally embedded and blocked in paraffin. Sections of $5 \mu \mathrm{m}$ were cut and stained with hematoxylin-eosin stain for measurement of epidermal thickness, toluidine blue stains for mast cell quantification, and trichrome stain for collagen visualization. Immunohistochemistry was performed using antitryptase and anti-IL-31 antibodies for characterizing mast cell degranulation and IL-31 expression in itch model of atopic dermatitis, respectively. The slides for immunohistochemistry were blocked with horse serum (Vector Laboratories, USA) and incubated with various antibodies for $16 \mathrm{~h}$. The slides were then incubated with ImmPRESS HRP reagent containing anti-mouse IgG (Vector Laboratories) for $30 \mathrm{~min}$, followed with incubation for 20 min with AEC peroxidase substrate (Vector Laboratories). Histopathological changes were then examined under a light microscope (Germany).

\section{Western Blotting}

Protein was extracted using radioimmunoprecipitation assay (RIPA) buffer (Thermo Scientific). The protein lysates ( $40 \mu \mathrm{g})$ were 
loaded onto either 7, 10, or $12 \%$ mini-protean TGX precast gels (BIO-RAD, USA) and electrophoresed at $100 \mathrm{~V}$ for $1.5 \mathrm{~h}$. The blots were transferred onto Immobilon-P polyvinylidene fluoride (PVDF) membranes (USA) for $1 \mathrm{~h}$ at $100 \mathrm{~V}$. After the transfer, the membranes were blocked with $5 \%$ skim milk-tris-buffered saline (TBS) for $1 \mathrm{~h}$ and then incubated overnight at $4^{\circ} \mathrm{C}$ with either antibody for IL-31, phosphor-ERK, phosphor-JNK, phospho-p38,

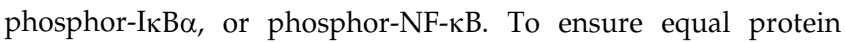
loading, the membranes were stripped and reprobed with anti- $\beta$ actin antibodies. Blots were incubated in respective secondary antibodies (Santa Cruz Biotechnology, USA) in 5\% skim milk-TBS for $2 \mathrm{~h}$ at room temperature and visualized on an ultraviolet detection Imaging System (Alliance version 15.11; UVITEC, UK). Band intensity was measured using the ImageJ gel analysis software (developed by the National Institutes of Health, Bethesda, USA).

Ribonucleic Acid (RNA) Extraction and Reverse TranscriptionPolymerase Chain Reaction (RT-PCR) Analysis

Total RNA was extracted and purified from excised dorsal skin using the Ribospin II Extraction Kit (GeneAll Biotechnology, Korea). The extraction was done following the manufacturer's specifications and the RNA extracted was stored at $-20^{\circ} \mathrm{C}$. The RNA $(1 \mu \mathrm{g})$ was reverse transcribed to cDNA using a PrimeScript RT Master Mix (Takara Bio Inc, Japan) following the manufacturer's protocol with a T100TM Bio-Rad Thermal Cycler set at $42^{\circ} \mathrm{C}$ for $1 \mathrm{~h}$. cDNA was amplified with Stratagene Mx3000P (Agilent Technologies Inc., USA) using the SYBR Premix Ex Taq (Takara Bio Inc., Japan) following the manufacturer's procedure. The standard cycle settings were: $95^{\circ} \mathrm{C}$ for $5 \mathrm{~min}, 40 \times\left(95^{\circ} \mathrm{C}\right.$ for $30 \mathrm{sec}$, $62^{\circ} \mathrm{C}$ for $30 \mathrm{sec}$ ) followed by a dissociation curve ramping from $95^{\circ} \mathrm{C}$ to $55^{\circ} \mathrm{C}$ and back to $95^{\circ} \mathrm{C}$. The following primers were used: sense, 5'-CCT ACC CTG GTG CTG CTT TG- $3^{\prime}$ and antisense, $5^{\prime}$ CTG ACA TCC CAG ATG CCT GC-3' for IL-31 ; sense, 5'-CCA GGA GCC ATA TCC ACG GA-3' and antisense, 5'-CTG TGA GGA CGT TTG GCA CA- $3^{\prime}$ for IL- 4 ; sense, $5^{\prime}$-GGC TAC ACT GAG GAC CAG GT-3' and antisense, 5'-TCC ACC ACC CTG TTG CTGTA-3' for glyceraldehyde 3-phosphate dehydrogenase (GAPDH). All samples were run in triplicate and fluorescence data of target genes were analyzed for relative quantification using GAPDH as a control.

\section{Enzyme-Linked Immunosorbent Assay (ELISA)}

On day 16 serum samples were collected and stored at $-20^{\circ} \mathrm{C}$. The samples were used in measuring the levels of IL-4 and IgE, using ELISA. The measurements were performed according to the manufacturer's protocol. The absorbance was immediately read at $450 \mathrm{~nm}$ on a spectrophotometer reader (Switzerland).

\section{Statistical Analysis}

Data are presented as the mean $\pm \operatorname{SD}(n=5)$. Statistical analysis was performed using one-way ANOVA followed by Duncan's multiple comparison tests. The $p$-values of $<0.05$, were considered statistically significant.

\section{Results}

SFBA Ameliorates Clinical Symptoms of Atopic Dermatitis

At the end of the 16-day treatment course, the anti-AD potentials of SFBA were evaluated by measuring the SCORAD clinical scores of $A D$, and the thickness of the excised dorsal skin. As shown in Fig. 1, repeated application of DNFB + house dust mite antigens led to an induction of AD-like symptoms in this study. The severity of the skin lesions was significantly reduced with oral administration of SFBA to the AD mice (Fig. 1B). Also, there was a significant reduction in the SCORAD clinical scores of atopic dermatitis (dryness, erosion excoriation oozing, and crust) in mice treated with SFBA (Fig. 1C). In addition, treatment with SFBA also led to a significant reduction in the dorsal skin thickness in the mice AD (Fig. 1D). To further analyze the efficacy of SFBA, we microscopically examined the dorsal skin sections of the mice. We confirmed that DNFB + house dust mite antigen challenge markedly induced epidermal hyperplasia and oral administration of SFBA prevented epidermal hyperplasia (Fig. 2A). Prednisolone, a positive standard used in this study showed similar results with SFBA.

\section{SFBA Prevented Infiltration of Mast Cells in the Skin of the Atopic Dermatitis-Mice Model}

In the present study, repetitive application of DNFB and house dust mite antigen induced enormous infiltration of mast cells into the dermis of the dorsal skin of the mice. However, when the AD model mice were treated with SFBA, marked suppression of mast cell infiltration into the dermis of the dorsal skin of the mice was observed (Fig. 2). As expected, prednisolone demonstrated similar results in preventing mast cell infiltration and degranulation in the dermis of the dorsal skin of the mice.

\section{SFBA Suppressed the Production of Interleukin-4 in Atopic Dermatitis-Mice Model}

The effectiveness of SFBA was also investigated in the present study by measuring the TH2-specific cytokine, IL-4. Figs. $3 \mathrm{~A}$ and $3 \mathrm{~B}$ reveal that the $\mathrm{AD}$ model group expressed the highest levels of IL-4 mRNA and IL-4 in the skin and serum respectively, compared with the normal control group. In contrast, the AD model group, co-administered with SFBA, considerably suppressed the expression of IL-4 

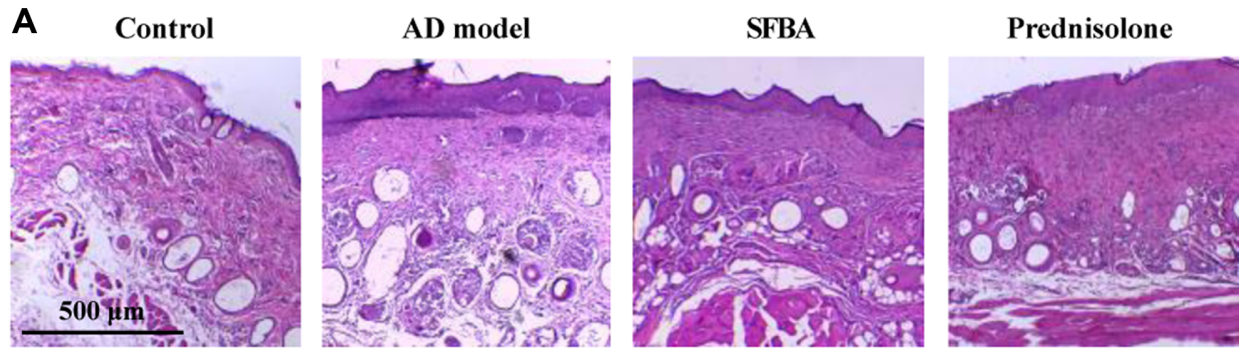

B
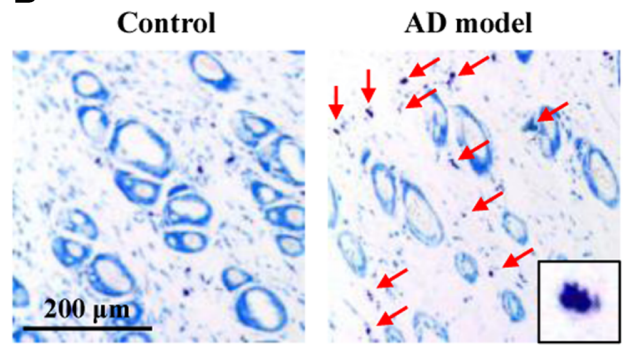

SFBA

Prednisolone
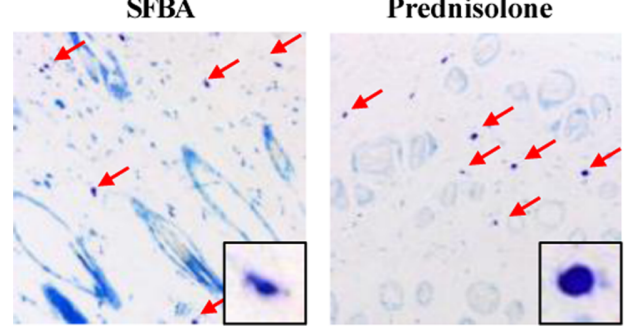

C

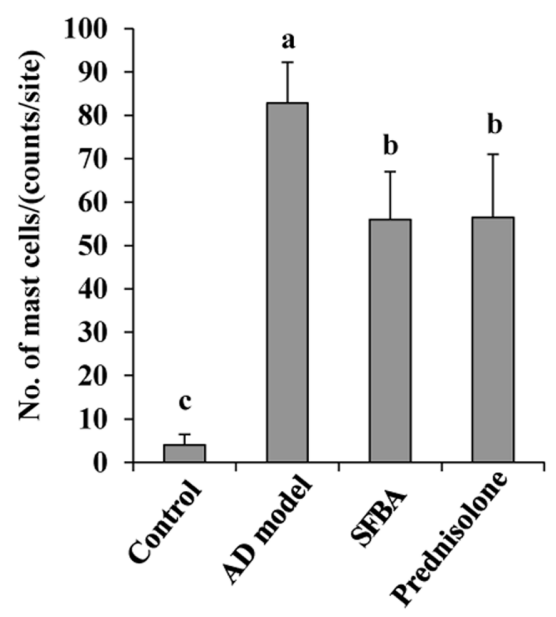

Fig. 2. Effect of SFBA treatment on epithelial hyperplasia and mast cell infiltration in the dermis of the atopic dermatitis mouse model.

(A) Morphological changes of dorsal skin stained with hematoxylin/eosin stain showing epithelial hyperplasia. (B and C) Toluidine blue stained tissues showing mast cell infiltration into the dermis. Images were taken at 10x magnification. Data are presented as the mean \pm SD $(n=5)$. Bars with different letters indicate statistically significant difference at $p<0.05$.

mRNA in the skin tissues and IL- 4 in the serum of the mice. Prednisolone also suppressed IL-4 mRNA expression in the dorsal skin and IL-4 in the serum of the mice.

\section{SFBA Suppressed the Expression of Immunoglobulin E in the Atopic Dermatitis-Mice Model}

The expression of IgE in mice serum was evaluated in this study. As shown in Fig. 3C, higher levels of IgE was found in mice serum applied with DNFB + house dust mite antigens. In contrast, oral administration of SFBA suppressed $\mathrm{IgE}$ expression in the serum. Also, oral administration of prednisolone slightly suppressed $\operatorname{IgE}$ expression in the mice serum.

\section{SFBA Suppressed Expression of Interleukin-31 in Atopic Dermatitis-Mice Model}

The efficacy of SFBA was also investigated in the present study by measuring the itch-related inflammatory cytokine,
IL-31. Figs. 3D and 3E reveal that the AD mice model group expressed the highest levels of IL-31 mRNA and proteins, compared with the normal control group. In contrast, the AD model group, co-administered with SFBA, considerably suppressed the expression of IL-31 mRNA and protein in the mice skin tissues. Prednisolone also suppressed the expression of IL-31 mRNA and protein in the mice skin tissues as expected.

\section{SFBA Significantly Reduced Itch in Atopic Dermatitis Itch-Mice Model}

The effect of SFBA on the relief of itch in ICR mice was investigated in the study. Injection of compound 48/80 on the dorsal skin of the mice induced scratching behavior in the neck of the mice. This scratching behaviour was significantly reduced in the mice treated with SFBA or prednisolone (Fig. 4F). Further histopathological examinations of tissue-stained sections showed a reduction of mast cell 

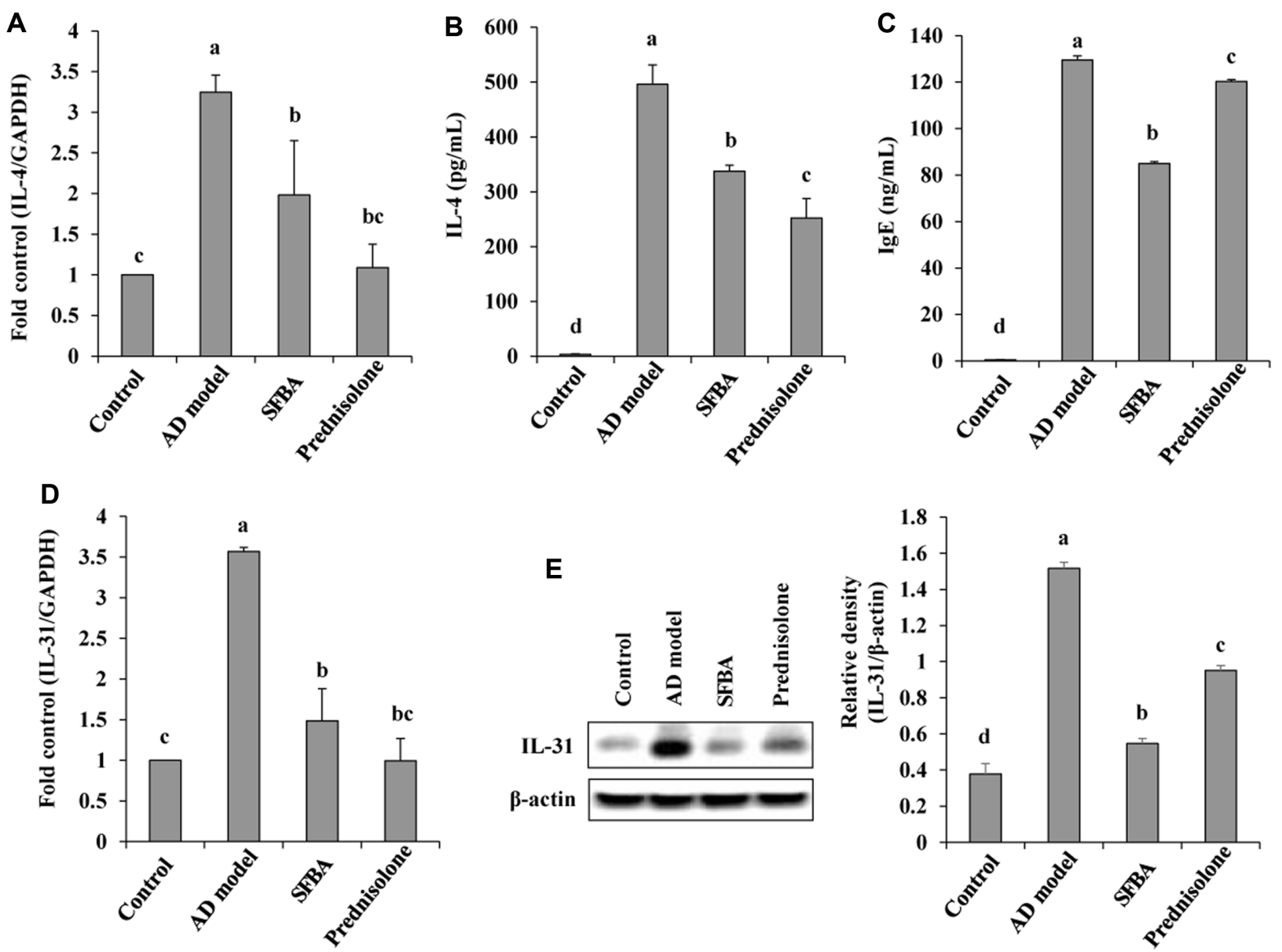

Fig. 3. Effect of treatment with SFBA on T helper cytokine, Immunoglobulin $\mathrm{E}$ and the itch-related interleukin-31 cytokine inhibition in the mouse model of atopic dermatitis.

(A) Dorsal skin tissue samples were collected and RT-PCR employed to determine the expression levels of IL-4 mRNA. (B and C) Serum samples were collected and used to measure IL-4 and IgE by ELISA. (D and E) RT-PCR and western blot analyses were employed to measure mRNA and the protein expressions levels of IL-31 in the dorsal skin tissues, respectively. Where applicable, data are presented as the mean \pm SD $(n=5)$. Bars with different letters indicate statistically significant difference at $p<0.05$.

infiltration and degranulation as evidenced by the decrease in the expression of tryptase in the dorsal skin of the mice treated with SFBA (Figs. 4B, 4C, and 4E). Also, the expression of IL-31 in the skin of the mice treated with SFBA was found to be decreased (Fig. 4D). As expected, prednisolone demonstrated similar effects with SFBA.

\section{SFBA Suppressed Collagen Deposition in Atopic Dermatitis- Mice Model}

In this study, processed skin specimens were also stained with Masson's trichrome to explore histological changes produced in collagen fibers. Results obtained are shown in Fig. 5 as photomicrographs representing the degree of collagen scaffold deposition in the dorsal dermis. DNFB + house dust mite antigens led to massive collagen deposition in the dermis. This showed that repeated topical applications of DNFB + house dust mite antigens that led to fibrogenesis with elevated production and deposition of collagen fibers in the dermal layers. However, when the mice induced with AD were repeatedly treated with SFBA, there was a considerably lower degree of collagen fiber deposition in the dorsal skin dermis. Prednisolone also prevented collagen deposition in the dermal skin.

SFBA Suppressed the Activation of Cell Signaling Molecules in the Skin Tissue of Atopic Dermatitis-Mice Models

To understand the mechanism of action of SFBA in 
A
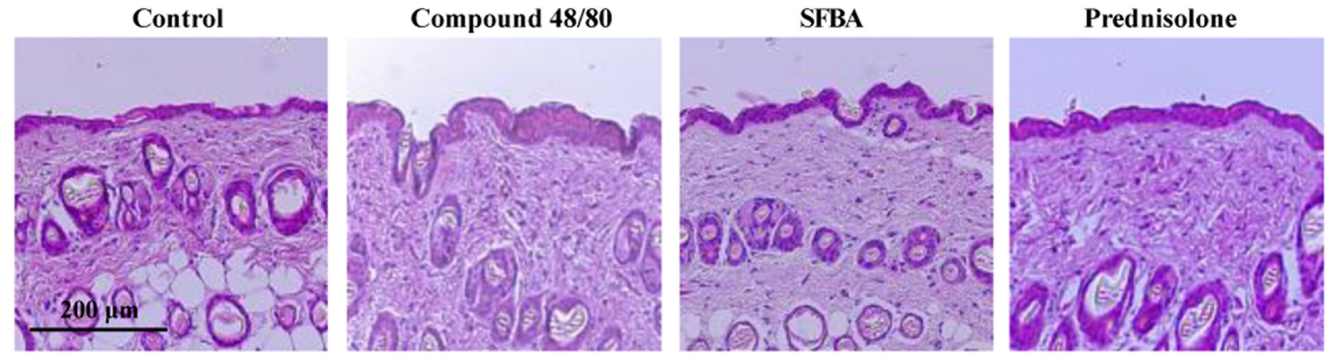

B
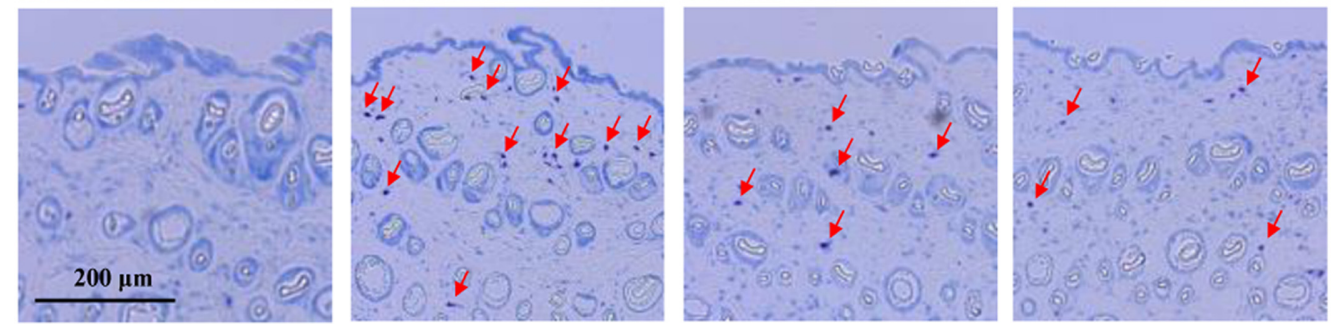

C
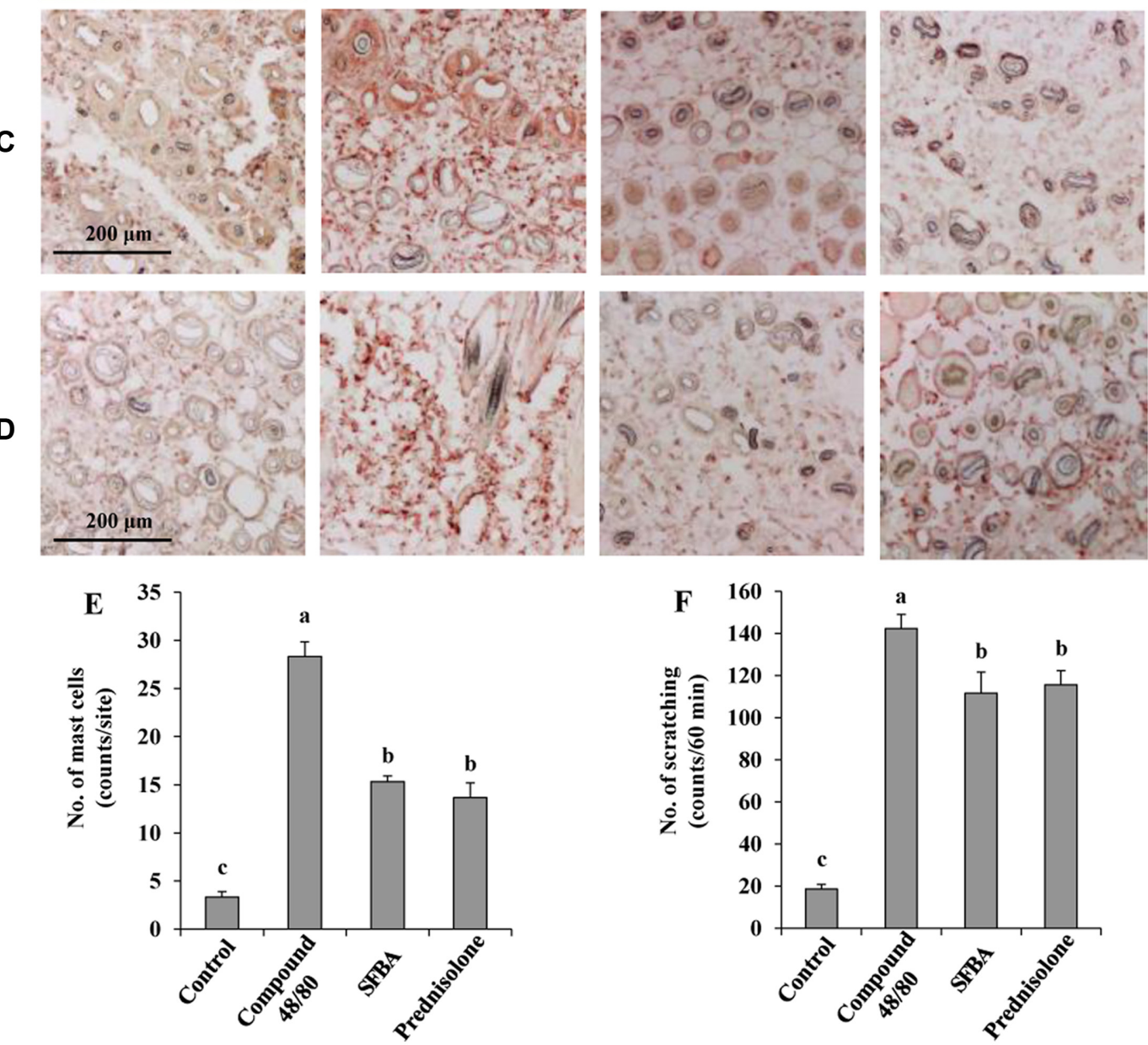

Fig. 4. Effect of treatment with SFBA on scratching behavior in compound 48/80-induced itch in ICR mice.

(A) Morphological changes of skin stained with hematoxylin/eosin stain. (B) Toluidine blue stained tissues showing mast cell degranulation. (C) Immunohistohemically stained tissues showing tryptase, a marker of mast cell degranulation. (D) Immunohistohemically stained tissues showing IL-31 expression in mice skin tissue. (E) The number of mast cells in the dermis was counted. (F) The number of scratches was recorded. Images were taken at $10 \times$ magnification. Data are presented as the mean \pm SD $(n=5)$. Bars with different letters indicated a statistically significant difference at $p<0.05$. 
A

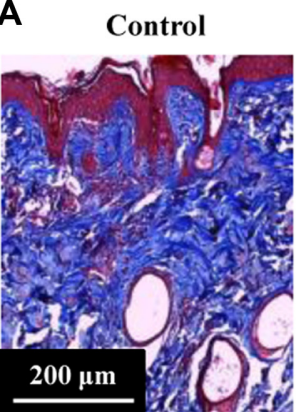

SFBA

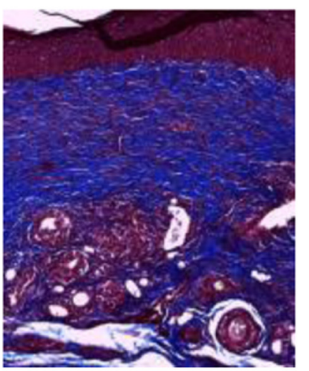

AD model

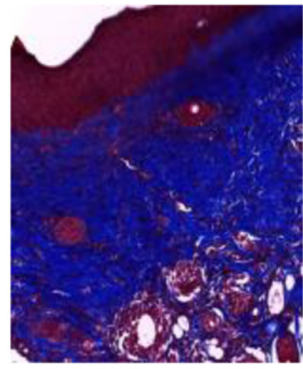

Prednisolone

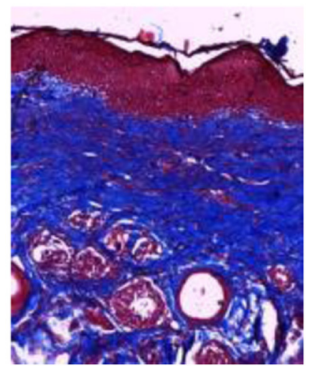

B

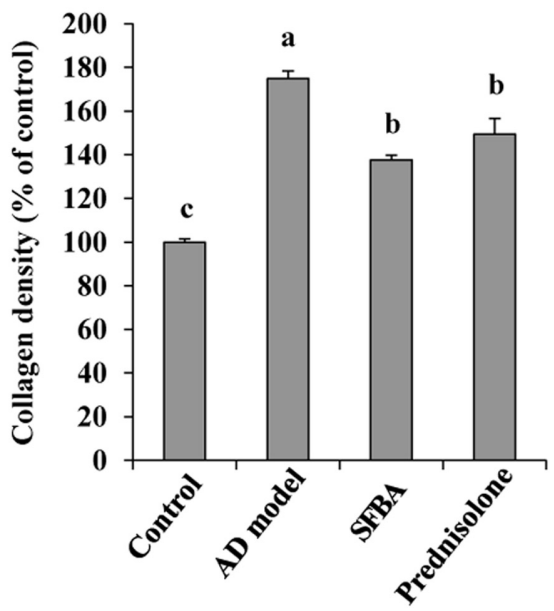

Fig. 5. Effect of treatment with SFBA on collagen fiber deposition in the dermis of the mouse model of atopic dermatitis.

(A) Representative trichrome stain for collagen fiber deposition. (B) The color density of trichrome stain-collagen fibers was measured with the help of Adobe Photoshop CS6 software and the results were quantified. Data are presented as the mean $\pm \operatorname{SD}(n=5)$. Bars with different letters indicated a statistically significant difference at $p<0.05$.

ameliorating the signs and symptoms of the $\mathrm{AD}$, the activation states of some key cell signaling molecules that are involved in directing the expressions and productions of AD-related cytokines were investigated. The results obtained showed that mice in the $\mathrm{AD}$ mice model group

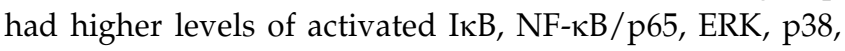
and JNK (denoted by their phosphorylated state), compared with the baseline group. Administration of SFBA to mice in the AD model group significantly suppressed the phosphorylation activation of all these signalling molecules in the mice skin (Fig. 6).

\section{Discussion}

Recently, as different treatment methods are proving ineffective for the treatment of $\mathrm{AD}$, scientists have begun to focus their interest on the development of alternative therapies for the treatment of atopic dermatitis which constitutes a nuisance to humans. In the present study, the preventive effectiveness of SFBA using a hairless mouse model of $\mathrm{AD}$ was explored by examining various $\mathrm{AD}$ related parameters including $\mathrm{AD}$ severities and signaling mechanisms responsible for the induction of $\mathrm{AD}$-related cytokines. The increased clinical scores and skin thickness observed in the study were expected to be caused by the activation of underlying inflammatory cascades associated with $\mathrm{AD}$ pathogenesis. These inflammatory reactions provoke various pathological processes, including accumulation of inflammatory cytokines in the dermis, epithelization, and keratinization. The results revealed that daily oral administration of SFBA did not only significantly reduce the clinical scores of $\mathrm{AD}$ but a significant reduction in the dorsal skin thickness and epidermal thickness were also recorded. In addition, SFBA prevented various pathological features of the AD such as severe erythema, hemorrhage, the release of exudates/transudates, edema, superficial erosion, and dry skin as seen in the obtained images. The results obtained for SFBA treatment were comparable to that obtained for prednisolone treatment. Therefore, these findings suggested that SFBA was effective in maintaining skin integrity distorted by the $A D$, and were associated with minimal symptoms of dryness and erythema.

AD is characterized by infiltration of inflammatory cells into the dermis of the skin. This was true for this study as the AD mouse model showed a large number of inflammatory mast cells in the dermis. The infiltration of mast cells to the dermis of the dorsal skin was in response 
A
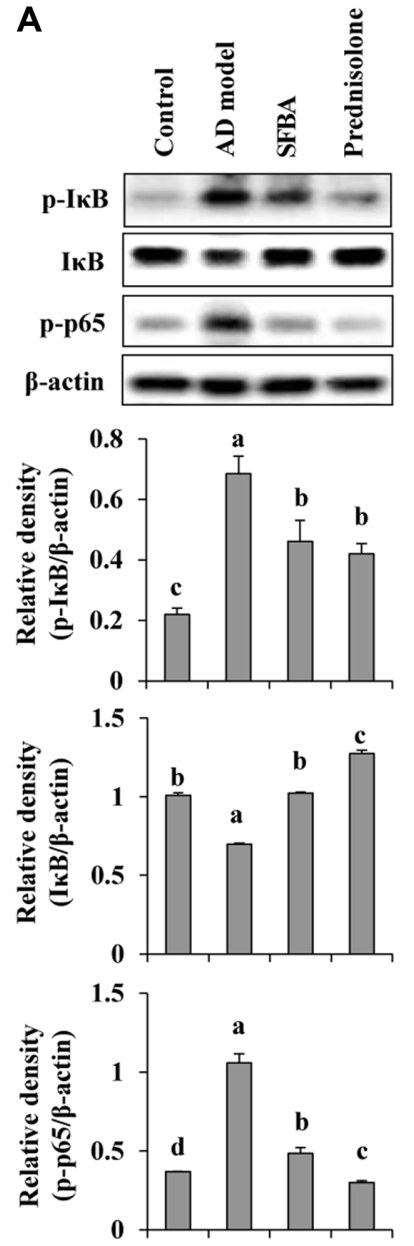

B
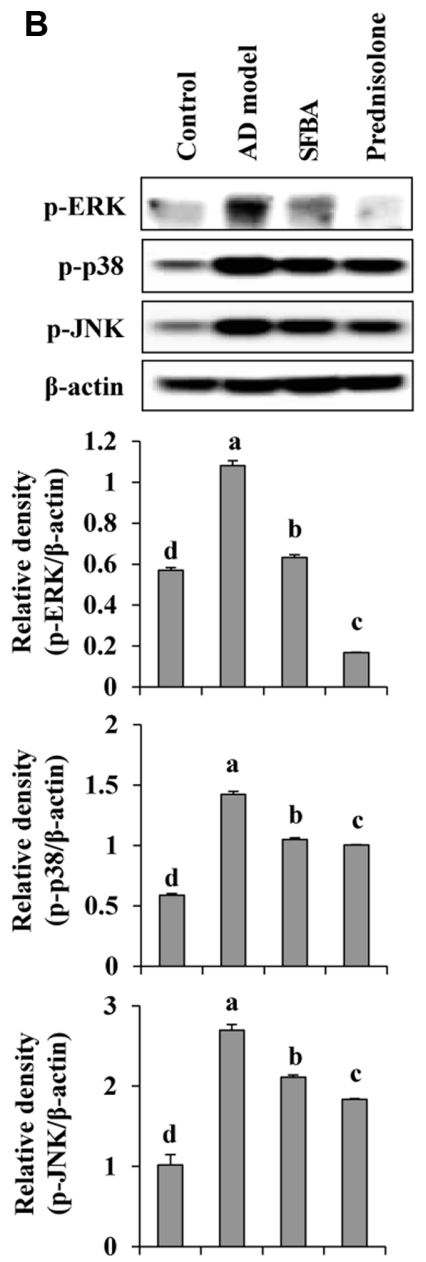

Fig. 6. Western blot analyses showing the effect of SFBA on the phosphorylation status of various cell signaling proteins in the skin of atopic dermatitis mouse model.

Proteins from the tissues were electrophoresed by SDS-PAGE, blotted onto a PVDF membrane and incubated with respective primary and secondary antibodies. The membranes were visualized using ECL western blotting substrate from Promega or Super Signal Western Dura Extended Duration Substrate from Thermo Scientific. The density of each band in the immunoblot was analyzed using the ImageJ gel analysis software. Bars with different letters indicated a statistically significant difference at $p<0.05$.

to the allergic inflammatory reaction in the skin caused by the repeated applications of DNFB and house dust mite antigen. In contrast, AD-induced mice treated with SFBA, like prednisolone, showed remarkable control of inflammatory mast cell infiltration into the dermis of the dorsal skin. The TH2-specific cytokine-IL-4 was also measured and found to be highly expressed in the skin tissues and serum of AD mice in the current study. Also,

IgE was found to be increased in the atopic mice group. This was in accordance with other studies that reported a high level of IgE in atopic mice that was due to activation of underlying inflammatory cascades in response to repetitive applications of DNFB [23, 24]. IL-4 is a key player in atopic dermatitis that signals through IL-4 receptor alpha and activates TH2 cells to further release IL-4, 5, 6, and 13 expressed in atopic dermatitis [25]. IL-4 also mediates the production of IgE in B cells during maturation. IgE then mediates the infiltration and degranulation of the mast cell that causes further infiltration of other inflammatory cells that are associated with AD [26]. Remarkably, our study showed that daily oral administration of SFBA to mice induced with AD minimized IL-4 and IgE production. The inhibitory effect of SFBA on IL-4 and IgE could be attributable to the action of SFBA to mitigate the progression of the adaptive immune response involved in $\mathrm{AD}$.

Itch is another symptom of $\mathrm{AD}$ with a mechanism that has been poorly understood. For this reason, treating itch in $\mathrm{AD}$ has proven difficult especially with the use of just histamine receptor blockers. Recently, a newly discovered cytokine, IL-31, has been found to be one of the sole mediators of itch in AD. Suppressing or eliminating IL-31 in $\mathrm{AD}$ patients could be beneficial in treating itch in $\mathrm{AD}$ [27]. Therefore, in the present study, we also investigated the effects of oral administration of SFBA in AD mouse models. Repetitive administration of DNFB and house dust mite antigen led to an increase in IL-31 mRNA and protein expression in the dorsal skin of mice in this study, similar to a report from a previous study [28]. This increase in IL$31 \mathrm{mRNA}$ and protein expression was suppressed when the $\mathrm{AD}$ mice were treated with SFBA, and the effect was comparable in magnitude to mice treated with prednisolone. The fact that SFBA decreased the expression of IL-31 in the mice skin pushed us to investigate the effects of SFBA on scratching behavior in mice subcutaneously administered with compound 48/80. Compound 48/80 activates mast cells in mice skin to release not only histamine but also other mediators including IL-31, which directly activate nerve fibers, thus inducing itch [29]. Compound 48/80 in this study indeed increased mast cell infiltration and degranulation as evidenced by the increase in the expression of tryptase, and increased the expression of IL31 in the skin of the mice. There was also an increase in scratching behavior induced by compound 48/80. However, SFBA pre-treatment significantly inhibited the infiltration and degranulation of mast cells, IL-31 expression, and scratching behavior in the mice. This protective effect was comparable in magnitude to the mice treated with 
prednisolone. Thus, it can be inferred that SFBA can reduce itch in AD mice by inhibiting the infiltration of mast cells into the dermis of the skin and expression of IL-31 in the skin tissue of mice.

Collagen deposition had previously been reported in atopic dermatitis mice [30]. Also in this study, an increase in collagen deposition was recorded. This could be as a result of the repeated topical applications of DNFB and house dust mite antigen that led to fibrogenesis with increasing production and deposition of collagen in the dermis. Lower collagen deposition was observed when the mice were administered with SFBA. The results were similar to the mice administered with prednisolone. The lowered collagen deposition might have contributed to improving the clinical scores reported for this study as decreased fibrogenesis and hence lower collagen deposition reduces skin fibrosis and scar formation. It can be suggested that the effect of SFBA on collagen deposition might be a result of its action in alleviating underlying inflammatory reactions involved in the initiation of fibrogenesis.

To understand the mechanism of action of SFBA in ameliorating DNFB and house dust mite-induced AD, we verify the phosphorylation status of signalling molecules that mediate inflammatory mediators and cytokine production in immune cells. Here, we found that DNFB and house dust mite antigen up-regulated the phosphorylation activation of ERK, p38, and JNK, IкB- $\alpha$, and NF- $\mathrm{KB}$, and oral administration of SFBA remarkably suppressed the phosphorylation activation of these signalling molecules in the mice dorsal skin. Prednisolone demonstrated similar effects with SFBA. These results suggest that SFBA suppress TH2-IL-4 and itch-related IL31 cytokine via suppressing I $\kappa \mathrm{B} \alpha$ and NF- $\mathrm{NB}$ activation as well as suppressing the activation of MAPKs (p38, ERK1/2, and JNK) in a mouse model of $\mathrm{AD}$, which offers a potential mechanism for the amelioration of the AD-like symptoms in mice.

In summary, our data, to the best of our knowledge showed for the first time that oral administration of SFBA ameliorated AD symptoms induced by DNFB and house dust mite antigens in hairless mice model of AD. SFBA was able to improve SCORAD scores of $A D$, reduce dorsal skin thickness, prevent collagen deposition in the dorsal skin, and reduce the infiltration and degranulation of mast cells, scratching behavior and expression of IgE, IL-4, and IL-31 through its prevention of NF-KB and MAPK activation in mice skin tissue. SFBA may serve as an effective food for preventing $\mathrm{AD}$ and inhibiting its progression.

\section{Acknowledgments}

This research was supported by the Traditional Culture Convergence Research Program through the National Research Foundation of Korea (NRF) funded by the Ministry of Science and ICT (NRF-2016M3C1B5907049).

\section{Conflict of Interest}

The authors have no financial conflicts of interest to declare.

\section{References}

1. Brandt EB, Sivaprasad U. 2011. Th2 cytokines and atopic dermatitis. J. Clin. Cell. Immunol. 2: pii:110.

2. Odhiambo JA, Williams HC, Clayton TO, Robertson CF, Asher MI. 2009. Global variations in prevalence of eczema symptoms in children from ISAAC phase three. J. Allergy Clin. Immunol. 124: 1251-1258.

3. Novak N. 2012. An update on the role of human dendritic cells in patients with atopic dermatitis. J. Allergy Clin. Immunol. 129: 879-886.

4. Ferretti E, Corcione A, Pistoia V. 2017. The IL-31/IL-31 receptor axis: general features and role in tumor microenvironment. J. Leukoc. Biol. 102: 711-717.

5. Howell MD, Kim BE, Gao P, Grant AV, Boguniewicz M, DeBenedetto A, et al. 2009. Cytokine modulation of atopic dermatitis filaggrin skin expression. J. Allergy Clin. Immunol. 124: R7-R12.

6. Kim BE, Leung DY, Boguniewicz M, Howell MD. 2008. Loricrin and involucrin expression is down-regulated by Th2 cytokines through STAT-6. Clin. Immunol. 126: 332-337.

7. Liu FT, Goodarzi H, Chen HY. 2011. IgE, mast cells, and eosinophils in atopic dermatitis. Clin. Rev. Allergy Immunol. 41: 298-310.

8. Nobbe S, Dziunycz P, Muhleisen B, Bilsborough J, Dillon SR, French LE, et al. 2012. IL-31 expression by inflammatory cells is preferentially elevated in atopic dermatitis. Acta Derm. Venereol. 92: 24-28.

9. Takaoka A, Arai I, Sugimoto M, Yamaguchi A, Tanaka M, Nakaike S. 2005. Expression of IL-31 gene transcripts in NC/Nga mice with atopic dermatitis. Eur. J. Pharmacol. 516: 180-181.

10. Toda M, Leung DY, Molet S, Boguniewicz M, Taha R, Christodoulopoulos $\mathrm{P}$, et al. 2003. Polarized in vivo expression of IL-11 and IL-17 between acute and chronic skin lesions. J. Allergy Clin. Immunol. 111: 875-881.

11. Shin JU, Kim SH, Kim H, Noh JY, Jin S, Park CO, et al. 2016. TSLP is a potential initiator of collagen synthesis and an ctivator of CXCR4/SDF-1 axis in keloid Pathogenesis. J. Invest. Dermatol. 136: 507-515. 
12. Dajee M, Muchamuel T, Schryver B, Oo A, Alleman-Sposeto J, De Vry CG, et al. 2006. Blockade of experimental atopic dermatitis via topical NF-кB Decoy Oligonucleotide. J. Invest. Dermatol. 126: 1792-1803.

13. Tanaka A, Muto S, Jung K, Itai A, Matsuda H. 2007. Topical application with a new NF- $\mathrm{KB}$ inhibitor improves atopic dermatitis in NC/NgaTnd mice. J. Invest. Dermatol. 127: 855863.

14. Johansen C, Kragballe K, Westergaard M, Henningsen J, Kristiansen K, Iversen L. 2005. The mitogen-activated protein kinases p38 and ERK1/2 are increased in lesional psoriatic skin. Br. J. Dermatol. 152: 37-42.

15. Su CL, Wu CJ, Chen FN, Wang BJ, Sheu SR, Won SJ. 2007. Supernatant of bacterial fermented soybean induces apoptosis of human hepatocellular carcinoma Hep 3B cells via activation of caspase 8 and mitochondria. Food Chem. Toxicol. 45: 303314.

16. Nakajima N, Nozaki N, Ishihara K, Ishikawa A, Tsuji H. 2005. Analysis of isoflavone content in tempeh, a fermented soybean, and preparation of a new isoflavone-enriched tempeh. J. Biosci. Bioeng. 100: 685-687.

17. Soh J, Kwon DY, Cha YS. 2011. Hepatic gene expression profiles are altered by dietary unsalted korean fermented soybean (chongkukjang) consumption in mice with dietinduced obesity. J. Nutr. Metab. 2011: 260214.

18. Kwon DY, Daily JW, 3rd, Kim HJ, Park S. 2010. Antidiabetic effects of fermented soybean products on type 2 diabetes. Nutr. Res. 30: 1-13.

19. Bae MJ, Shin HS, See HJ, Chai OH, Shon DH. 2014. Cheonggukjang ethanol extracts inhibit a murine allergic asthma via suppression of mast cell-dependent anaphylactic reactions. J. Med. Food 17: 142-149.

20. Choi YH, Lim H, Heo MY, Kwon DY, Kim HP. 2008. Antiinflammatory activity of the ethanol extract of Chungkukjang, Korean fermented bean: 5-lipoxygenase inhibition. J. Med. Food 11: 539-543.
21. Mihara K, Kuratani K, Matsui T, Nakamura M, Yokota K 2004. Vital role of the itch-scratch response in development of spontaneous dermatitis in NC/Nga mice. Br. J. Dermatol. 151: 335-345.

22. Cho BO, Che DN, Yin HH, Shin JY, Jang SI. 2017. Diospyros lotus leaf and grapefruit stem extract synergistically ameliorate atopic dermatitis-like skin lesion in mice by suppressing infiltration of mast cells in skin lesions. Biomed. Pharmacother. 89: 819-826.

23. Kang MJ, Eum JY, Park SH, Kang MH, Park KH, Choi SE, et al. 2010. Pep-1 peptide-conjugated elastic liposomal formulation of taxifolin glycoside for the treatment of atopic dermatitis in NC/Nga mice. Int. J. Pharm. 402: 198-204.

24. Matsuda H, Watanabe N, Geba GP, Sperl J, Tsudzuki M, Hiroi J, et al. 1997. Development of atopic dermatitis-like skin lesion with IgE hyperproduction in NC/Nga mice. Int. Immunol. 9: 461-466.

25. Philips N, Samuel M. 2017. Inhibition of Interleukin-4 signalling in the treatment of atopic dermatitis and allergic asthma. Glob. J. Allergy 3: 019-021.

26. Kishimoto T, Hirano T. 1988. Molecular regulation of B lymphocyte response. Annu. Rev. Immunol. 6: 485-512.

27. Wong LS, Wu T, Lee C-H. 2017. Inflammatory and noninflammatory itch: implications in pathophysiologydirected treatments. Int. J. Mol. Sci. 18: 1485.

28. Jin H, He R, Oyoshi M, Geha R. 2009. Animal models of atopic dermatitis. J. Invest. Dermatol. 129: 31-40.

29. Ribeiro-Filho J, Leite FC, Costa HF, Calheiros AS, Torres RC, de Azevedo CT, et al. 2014. Curine inhibits mast cell-dependent responses in mice. J. Ethnopharmacol. 155: 1118-1124.

30. Hussain Z, Katas H, Mohd Amin MCI, Kumolosasi E. 2014. Efficient immuno-modulation of $\mathrm{T}(\mathrm{H} 1) / \mathrm{T}(\mathrm{H} 2)$ biomarkers in 2,4-dinitrofluorobenzene-induced atopic dermatitis: nanocarriermediated transcutaneous co-delivery of anti-inflammatory and antioxidant drugs. PLoS One 9: e113143. 